\title{
Programa escolar de educación financiera Sernac
}

\author{
Sernac financial education school program \\ Paula Bustos
}

\section{Introducción}

Hoy nuestros niños y niñas están insertos en el mundo del consumo desde temprana edad, por lo que es necesario que desde su etapa escolar puedan contar con herramientas que les permitan enfrentar situaciones de consumo como ciudadanos empoderados, y críticos, con la información y competencias necesarias para tomar decisiones correctas. La Educación Financiera permite que nuestros niños, niñas y jóvenes, puedan adquirir competencias contribuyendo a combatir el sobreendeudamiento a través de una gestión adecuada de las finanzas personales y familiares, entendiendo también que el mercado financiero es uno de los que presenta las mayores asimetrías de información, y que originan el mayor número de reclamos ante el Sernac.

Es por lo anterior, que desde el Departamento de Educación para el Consumo del Sernac, se trabaja en la Educación Financiera tanto en la educación formal como no formal, para hacer llegar a los niños, niñas y jóvenes de Chile herramientas para entender conceptos fundamentales como tasa de interés, ahorro, inversión, carga anual equivalente (CAE), costo total del crédito, entre otras.

En este contexto, el Sernac ha diseñado e implementado el Programa Escolar de Educación Financiera (PEEF), el cual tiene por objetivo formar ciudadanos críticos frente a las presiones del mercado, capaces de exigir sus derechos y tomar decisiones de consumo informadas y responsables en las distintas etapas de la vida. Asimismo, aportar en la formación de ciudadanos conscientes de la importancia de jerarquizar recursos y diferenciar deseos de necesidades, que logren visualizar el beneficio de generar ahorro y prevenir el sobreendeudamiento. Ciudadanos que, por lo demás, logren comprender que las decisiones individuales de consumo tienen un impacto en la colectividad y, en distintas áreas como el medio ambiente, la economía y el desarrollo social.

Entre los factores que desencadenan la preocupación por la Educación Financiera (EF) a nivel mundial encontramos el alto nivel de reclamos de

*Dirección de correspondencia [Correspondence address]: Paula Bustos, Universidad de Chile

E-mail: paula.bustos@gmail.com consumidores respecto al mercado financiero; el alto nivel de endeudamiento de las personas y su dependencia respecto al mundo financiero; la desinformación y falta de comprensión de los servicios financieros; el surgimiento de nuevas formas de manejar el dinero; el creciente envejecimiento de la población, que implica que las personas deben vivir más años con la pensión de jubilación que reciben; y la proliferación de una sociedad que le entrega gran importancia al consumo, incentivando el consumo a crédito y desincentivando el ahorro. Además, las generaciones más jóvenes tendrán que soportar mayores riesgos financieros y se enfrentarán a productos cada vez más complejos y sofisticados. Podemos observar que los jóvenes están teniendo acceso y recibiendo ofertas de servicios financieros cada vez a más temprana edad. En lo referente al conocimiento básico financiero, la Encuesta de Conocimiento Financiero del Sernac 2015 evidencia un alto analfabetismo en esta materia y una baja capacidad de aplicación de los conceptos para una correcta toma de decisiones.

En nuestro país existe preocupación en torno al funcionamiento del mercado financiero, ello a partir de algunas situaciones particulares que golpearon a la dinámica financiera nacional. Dos casos emblemáticos ocurridos durante el año 2011 encendieron una alerta en torno al tema; las prácticas de colusión para el alza de precios entre las tres principales cadenas de farmacias del país, y el escándalo que provocó el descubrimiento de repactaciones unilaterales realizadas por la multitienda La Polar a sus clientes, el que afectó a casi un millón de personas. Ambos casos alcanzaron gran cobertura mediática por la enorme cantidad de personas afectadas, escenario que puso en alerta a diferentes instituciones en torno a la posición en la que se encuentra la población en general. Respondiendo a situaciones como las recién descritas, el año 2012 se promulga la Ley $\mathrm{N}^{\circ} 20.555$. Esta es fundamental para el posicionamiento de la EF a nivel estatal, ya que fortalece en términos legales la protección de los derechos de los consumidores y regula los servicios y productos financieros. Este entramado de factores da paso a una creciente preocupación en torno a la falta de preparación de las personas y de las instituciones de los Estados con respecto al contexto financiero, surgiendo importantes iniciativas en torno a la Educación Financiera en distintos países. 
Frente al panorama mundial y las conocidas crisis financieras, la Organización para la Cooperación y el Desarrollo Económico (OCDE) es una de las primeras instituciones que levanta la voz y propone la EF como principal motor para enfrentar la situación antes descrita, y sostiene que esta ha de iniciarse lo antes posible en la vida de una persona. Señala el organismo que incluir Educación Financiera en el currículum formal es reconocido como una de las formas más eficientes y justas para llegar a toda una generación a gran escala, que es un medio único para inculcar y fomentar una adecuada cultura y comportamientos financieros, que los niños y jóvenes son buenos diseminadores de nuevos hábitos y que la experiencia respecto de aquellos niños y jóvenes que han recibido algún tipo de formación en finanzas personales en edades tempranas demuestra que es clave para desarrollar hábitos adecuados que permitan obtener el mejor beneficio del sistema financiero. Desde esta perspectiva, los procesos de educación financiera en la escuela se convierten en un elemento fundamental a considerar, ya que es en este espacio donde los estudiantes desarrollan conocimientos, competencias y actitudes iniciales que determinan su desarrollo económico posterior.

Conforme con esto, la OCDE ha definido la EF en el contexto de la escuela como la enseñanza de conocimientos financieros, comprensión, habilidades, comportamientos, actitudes y valores que permitirán a los estudiantes tomar decisiones financieras efectivas en su vida cotidiana y en su adultez (OCDE, 2012).

Dados los antecedentes internacionales y nacionales relacionados con el alto nivel de analfabetismo financiero, el sobreendeudamiento, y los lineamientos de la OCDE sobre la materia, el Sernac diseñó el año 2012 el Programa Escolar de Educación Financiera, dirigido a establecimientos Municipales y Particulares Subvencionados. Al año siguiente, el Ministerio de Educación decide incorporar contenidos de educación financiera en el currículum escolar, a través de la asignatura de Historia, Geografía y Ciencias Sociales para $1^{\circ}$ medio. Junto con ello, Chile se compromete a evaluar las competencias financieras de los estudiantes chilenos a través de la prueba PISA $^{1}$, desde el año 2015 en adelante.

También el año 2013, se crea la Comisión Asesora de la Presidenta de la República para la Inclusión Financiera, a cargo del Ministerio de Hacienda, la cual tiene como misión asesorar a la Presidenta en

\footnotetext{
${ }^{1}$ El Programa para la Evaluación Internacional de Alumnos de la OCDE (PISA, por sus siglas en inglés), tiene por objeto evaluar hasta qué punto los alumnos cercanos al final de la educación obligatoria han adquirido algunos de los conocimientos y habilidades necesarios para la participación plena en la sociedad del saber.
}

el diseño y formulación de una Estrategia Nacional de Inclusión Financiera. En ese sentido, el Programa Escolar de Educación Financiera propuesto es un complemento relevante para lograr los objetivos que se ha planteado el país y un apoyo fundamental para la comunidad escolar y las instituciones públicas.

La Educación Financiera, no sólo implica conocimientos sobre el mundo económico y operaciones matemáticas, implica también el desarrollo de competencias que permiten a los ciudadanos y ciudadanas actuar con propiedad en el mundo financiero del que son parte. Por otro lado, es fundamental recoger el conocimiento que tienen los niños y niñas sobre estas temáticas e incorporarlos a los procesos educativos, para que estos temas puedan ser aplicados desde la cotidianeidad de la experiencia.

El Programa está dirigido a distintos actores de la comunidad educativa, tales como directivos, docentes, estudiantes y apoderados. Sin embargo, su foco principal lo constituyen los y las estudiantes que se espera adquieran las competencias necesarias para desenvolverse de manera empoderada en el mundo financiero.

El Programa de EF, así como las acciones educativas que lo conforman, se enmarca en un enfoque socio constructivista del proceso de enseñanzaaprendizaje. De este modo, se busca que los y las estudiantes participen de manera activa en la construcción de sus propios aprendizajes, los que surgirán a partir de sus experiencias personales sobre el tema. En este contexto, el rol docente adquiere un rol central como mediador de las experiencias de aprendizaje de los estudiantes, incorporando el desarrollo de las temáticas de EF a partir de situaciones cotidianas.

\section{Dimensiones centrales del modelo de educación financiera escolar}

El modelo de Educación Financiera en la Escuela del Sernac, se diseñó a partir de las recomendaciones de la OCDE, de Child and Youth Financial International, Aflatoun, UNESCO y de la Universidad de la Frontera, a través de su Centro de Psicología Económica y Consumo. Este modelo, se compone de cuatro dimensiones centrales que se entrelazan y complementan entre sí: contenidos, contextos, procesos y valores y actitudes.

Respecto a los contenidos, existen cinco ejes temáticos del Programa de EF chileno: panorama financiero, derechos y deberes, dinero y su uso, planificación y elaboración de presupuestos y ahorro e inversión. En cuanto a los contextos se conside- 
ra que son distintas las situaciones en las que se aplican los conocimientos financieros, siendo fundamental que los niños, niñas y jóvenes puedan experimentar lo aprendido en diferentes escalas que van desde lo local a lo global. En ese sentido, el programa considera contextos personales, familiares, educacionales, laborales y sociales.

Los procesos indican el recorrido cognitivo que debería guiar el aprendizaje financiero. Los pasos que se consideran en este proceso son: Identificar información financiera; analizar la información en un contexto financiero; evaluar cuestiones financieras; y, aplicar el conocimiento financiero. Como todo proceso cognitivo de adquisición de aprendizajes, este recorrido debe ser evaluado en función del contexto y grado de complejidad desarrollado por los/as estudiantes con los que se trabaja.

Además, para el Sernac es fundamental introducir en cada uno de los contenidos la dimensión de "Valores y Actitudes" que propone la experta Marianela Denegri en su modelo de educación económica y financiera. Estos son: actitudes racionales hacia la compra; tolerancia al riesgo; sensibilidad temporal; locus de control interno; y, actitudes positivas hacia el consumo responsable (Denegri, 2010).

\section{Metodología}

La necesidad de generar transformaciones en las dinámicas financieras a partir del empoderamiento de la ciudadanía, implica enseñar EF de una forma particular que permita el cumplimiento de las metas propuestas. Para esto la metodología socio-constructivista, en coherencia con el modelo pedagógico que sustenta el Programa, permite orientar las acciones pedagógicas hacia el desarrollo de aprendizajes significativos, los que desarrollan transformaciones en las estructuras cognitivas de los/as estudiantes, logrando aprendizajes de largo alcance, duración y aplicabilidad.

Al respecto, es necesario visualizar tres principios metodológicos claves para su alcance: el desarrollo de competencias financieras que permitan un actuar consciente y responsable; el desarrollo de estrategias de enseñanza participativas que permitan el empoderamiento de los estudiantes; y la orientación hacia una mirada crítica de los servicios y productos financieros. Para incorporar estos principios metodológicos a las prácticas de enseñanza de EF se han establecido líneas de acción en torno a los elementos fundamentales del modelo socio-constructivista:

- Apropiación del Docente: Es fundamental que los docentes puedan familiarizarse con los con- tenidos de EF para hacer un tratamiento riguroso de los temas a trabajar. Esto significa no sólo conocer los ejes temáticos y las principales definiciones y conceptos centrales, sino visualizar el carácter valórico de cada uno de los ejes y del programa en general.

- Participación activa de los estudiantes: La participación activa de los y las estudiantes en las actividades de aprendizaje favorecerá el desarrollo de mejores y más significativos aprendizajes. Sin embargo, es importante mencionar que, más allá de "realizar muchas actividades" es fundamental que sean protagonistas, puedan opinar, se sientan partícipes de las actividades y se consideren incluidos y tomados en cuenta.

- Estrategias de Implementación curricular: Actualmente, la Educación Financiera no es una asignatura en nuestros programas de estudio, por lo cual debe enseñarse en contextos que permitan su incorporación a través de diferentes formas de tratamiento de sus contenidos y habilidades. En el caso de algunas asignaturas, como por ejemplo Historia, Geografía y Ciencias Sociales, existen contenidos de educación financiera asociados a conceptos económicos que forman parte del programa de dicha asignatura, razón por la cual, se puede establecer una relación directa entre contenidos financieros y Ciencias Sociales.

- Aquellas temáticas de Educación Financiera que no estén directamente explicitadas en los programas de estudio de la asignatura, pueden ser abordadas al interior de la sala de clases a través de la incorporación de textos escritos y/o mensajes de medios de comunicación que aborden contenidos de Educación Financiera. Además, en algunas asignaturas, los estudiantes desarrollan habilidades con la finalidad de ser aplicadas a distintos ámbitos de su vida cotidiana, es así como los contenidos de Educación Financiera pueden incorporarse en la sala de clases para afianzar por ejemplo habilidades de cálculo, razonamiento lógico, de organización de datos y otros.

\section{Elementos del programa de Educación Financiera}

Los elementos o componentes del programa son los siguientes:

- Curso de perfeccionamiento docente: Con el objetivo de aportar al perfeccionamiento de los 
y las docentes en materias de EF, el Sernac imparte el curso de perfeccionamiento e-learning Educación Financiera en la Escuela. Se imparte una vez al año, cuenta con tutoría especializada y está certificado por el CPEIP (Centro de Perfeccionamiento, Experimentación e Investigaciones Pedagógicas).

- Guías didácticas para docentes y estudiantes: Junto al Programa de EF, el Sernac pone a disposición de los profesores y profesoras una guía para el docente y una guía para el estudiante, las que contienen los elementos principales del Programa, recursos pedagógicos, junto con orientaciones metodológicas, ejemplos de actividades y glosario de términos.

- Talleres para trabajar con familias: Con el objetivo de facilitar la incorporación de familias al programa, el Sernac pone a disposición de padres y madres talleres para ser trabajados en espacios de reunión de apoderados o escuelas para padres. Estos talleres pueden ser solicitados a través del sitio web.

- Material educativo: El Sernac cuenta con diverso material educativo que puede ser utilizado por profesores y profesoras para el desarrollo de aprendizajes de educación financiera: entre ellos se encuentran videos y series audiovisuales, fichas con actividades pedagógicas, juegos y otros.

- Concurso de Educación Financiera: El Sernac realiza anualmente, a partir del 2014, un Concurso Escolar de Educación Financiera llamado Al Chancho, con el objetivo de promover conductas de consumo responsable en el ámbito financiero, como el ahorro y la planificación, dirigido a todos los establecimientos públicos y particulares subvencionados del país.

- Actividades educativas para niños, niñas y jóvenes: Con el objetivo de apoyar la incorporación de la educación financiera en el aula, el Sernac ofrece a los establecimientos actividades educativas presenciales, a modo de charlas y talleres, las que podrán ser solicitadas a través de su sitio web. También se ofrecen actividades como obras de teatro, concursos en vivo, actividades lúdicas, torneos, etc.

\section{El sistema de reconocimiento}

Si bien cada establecimiento educacional puede definir su propia manera de implementar la Educación Financiera en su comunidad, el Sernac, en conjunto con la Universidad de La Frontera, a través de su Centro de Psicología Económica y Consumo
$(\mathrm{CEPEC})^{2}$, promueven que los establecimientos escolares se incorporen al sistema de reconocimiento. El sistema permite la demostración confiable y verificable de que una comunidad educativa cumple con la incorporación de temas financieros en la escuela. Este reconocimiento es otorgado por quienes verificarán si los establecimientos cumplen con los requisitos para obtener nivel básico, medio o de excelencia en educación financiera.

\section{Participantes}

El Sistema de Reconocimiento de Establecimientos Educacionales en Educación Financiera es un sistema voluntario para todos los establecimientos de Enseñanza Básica y Enseñanza Media de Chile de dependencia municipal o particular subvencionado vulnerable. Cada establecimiento define a los cursos o niveles a los cuales postulará al Reconocimiento, pudiendo optar desde $6^{\circ}$ básico a $4^{\circ}$ medio en un inicio.

Para optar al Reconocimiento del Nivel Básico, luego de inscribirse vía web, el colegio debe difundir a la comunidad docente su incorporación al Programa; coordinar la aplicación de una prueba de diagnóstico; capacitar a sus docentes en Educación Financiera; y realizar actividades de educación financieras como charlas, obra de teatro, paseos a bancos, etc., con los estudiantes y sus familias.

El Reconocimiento del Nivel Medio, requiere que el colegio capacite a nuevos docentes; que realice nuevas actividades de educación financiera; que muestre un PEI comprometido con la educación financiera; ejecutar al menos 3 actividades de aprendizaje en 3 asignaturas diferentes; y realizar una actividad o un proyecto colectivo interno.

Por último, para lograr el Reconocimiento de Nivel de Excelencia, el colegio debe haber cumplido con los requisitos anteriores; someterse a la aplicación de una prueba de parte del Sernac; incorporar actividades pedagógicas en nuevas asignaturas y niveles educativos; y promover la educación financiera en una red comunitaria. En todo este periodo el colegio es asesorado y acompañado por profesionales del Sernac.

$\mathrm{Al}$ iniciar el proceso de certificación, los colegios

\footnotetext{
${ }^{2}$ El CEPEC es un Centro dependiente de la Facultad de Educación, Ciencias Sociales y Humanidades de la Universidad de La Frontera, especializado en investigación y consultoría en psicología económica, alfabetización económica y financiera, comportamiento del consumidor, comportamiento microempresarial y consumo responsable. Es dirigido por la Dra. Marianela Denegri Coria, precursora de la Psicología Económica Latinoamericana y una de las personalidades actuales más relevantes en la psicología social en Chile.
} 
obtienen una serie de beneficios, partiendo por el reconocimiento público por incorporar la educación financiera en la escuela y por promover prácticas de consumo responsable en su comunidad. Además puede acceder a cursos, talleres y reuniones de trabajo sobre la temática para docentes, directivos y otros integrantes de la comunidad escolar, a material educativo de apoyo, obras de teatro, torneos y a información sobre convocatorias a concursos y actividades de otras instituciones. Por último, puede contar con la presencia del Sernac en actividades y charlas para la comunidad escolar.

\section{Resultados}

El Programa Escolar de Educación Financiera comienza a diseñarse a fines de 2012 y en marzo del año siguiente, comienza con 30 colegios que se comprometen a incorporar la educación financiera en sus actividades curriculares y extracurriculares, involucrando a toda la comunidad educativa.

Desde ese año a la fecha, los principales avances en cuanto a generación de contenidos y productos refieren a la implementación de un curso de perfeccionamiento docente llamado "Educación financiera en la escuela", que se imparte 2 veces al año, a la definición de una red de contenidos, al diseño de fichas y guías para el trabajo docente, la elaboración de una serie de animaciones audiovisuales, de una obra de teatro sobre la temática, la guía del docente y la del estudiante. Además, se desarrolló un sitio web propio del programa (www.sernaceduca.cl) y se incorporaron establecimientos de todas las regiones del país.

En relación a resultados de cobertura, durante el 2014 el programa contó con 58 colegios inscritos, número que aumentó a 244 el 2015. Con lo anterior, se vislumbra impactar a, al menos, 72.000 estudiantes. Asimismo, en relación al curso de perfeccionamiento que dicta el Sernac en conjunto con el CPEIP, el año 2014 los docentes capacitados implementaron 425 transferencias al aula o clases de educación financiera a sus estudiantes, es decir, formaron en esta materia a 12.750 alumnos.

Por otra parte, es importante señalar que tanto a los docentes como a los estudiantes de los colegios que son parte del programa, se les aplicó una prueba de diagnóstico para determinar su nivel inicial de conocimiento en la materia. Respecto a los docentes los resultados indican una brecha importante en temáticas como: la misión de la AFP, qué es el flujo de producción, el principio de eficiencia en la toma de decisiones y significado del dinero como medida de valor.
No obstante, finalizado el curso de perfeccionamiento, se logró un avance significativo, mejorando sus niveles de conocimiento a nivel global (de un $68 \%$ de respuestas correctas a un $93 \%$ ) y en cada uno de los ejes de contenidos, como se observa en el siguiente gráfico:

En relación a los estudiantes, se presentan los resultados de 942 estudiantes de enseñanza media, lo cual si bien no es representativo, de todos modos presenta ciertas tendencias. Al respecto, destaca el bajo nivel de conocimiento en materias asociadas al ahorro y la inversión (sobre todo aquellas que requieren habilidades como el cálculo), la planificación financiera y elaboración de presupuesto, así como el conocimiento sobre derecho y deberes del consumidor.

A futuro, se espera observar los avances en conocimiento y competencias de los jóvenes al aplicarles nuevamente esta prueba, una vez hayan recibido clases y talleres sobre educación financiera. 
Figura 1: Nivel de Conocimiento Financiero prueba diagnóstica y final (Docentes que finalizan curso de perfeccionamiento del SERNAC; $\mathrm{N}=188$ )

\section{Porcentaje de respuestas correctas}

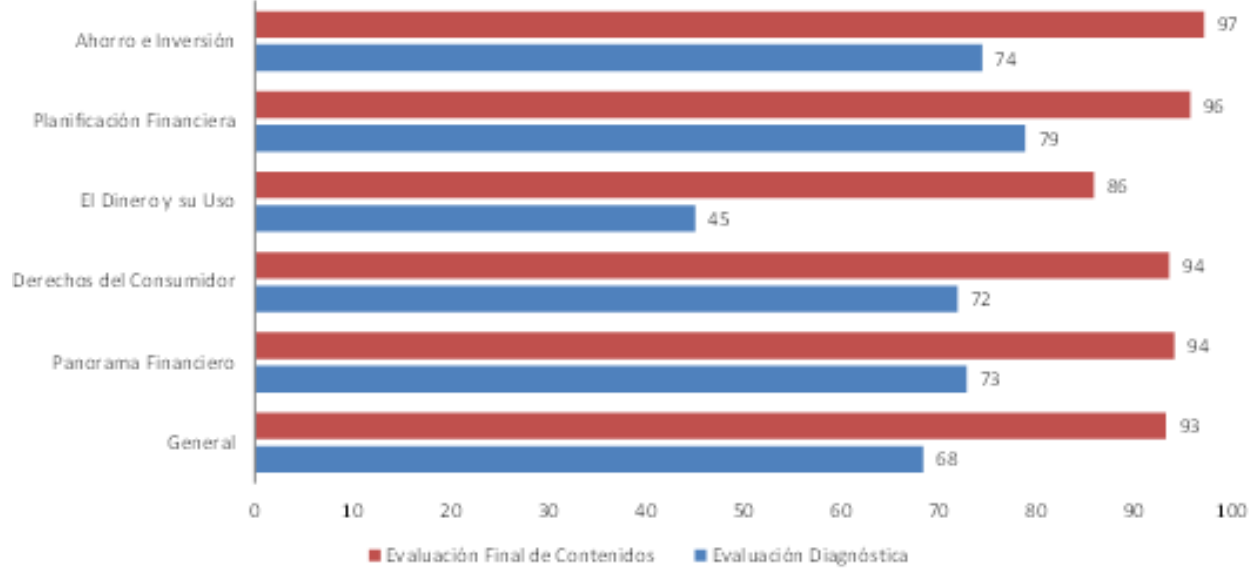

Fuente: Elaboración propia

Figura 2: Porcentaje de respuestas correctas según eje de contenidos)

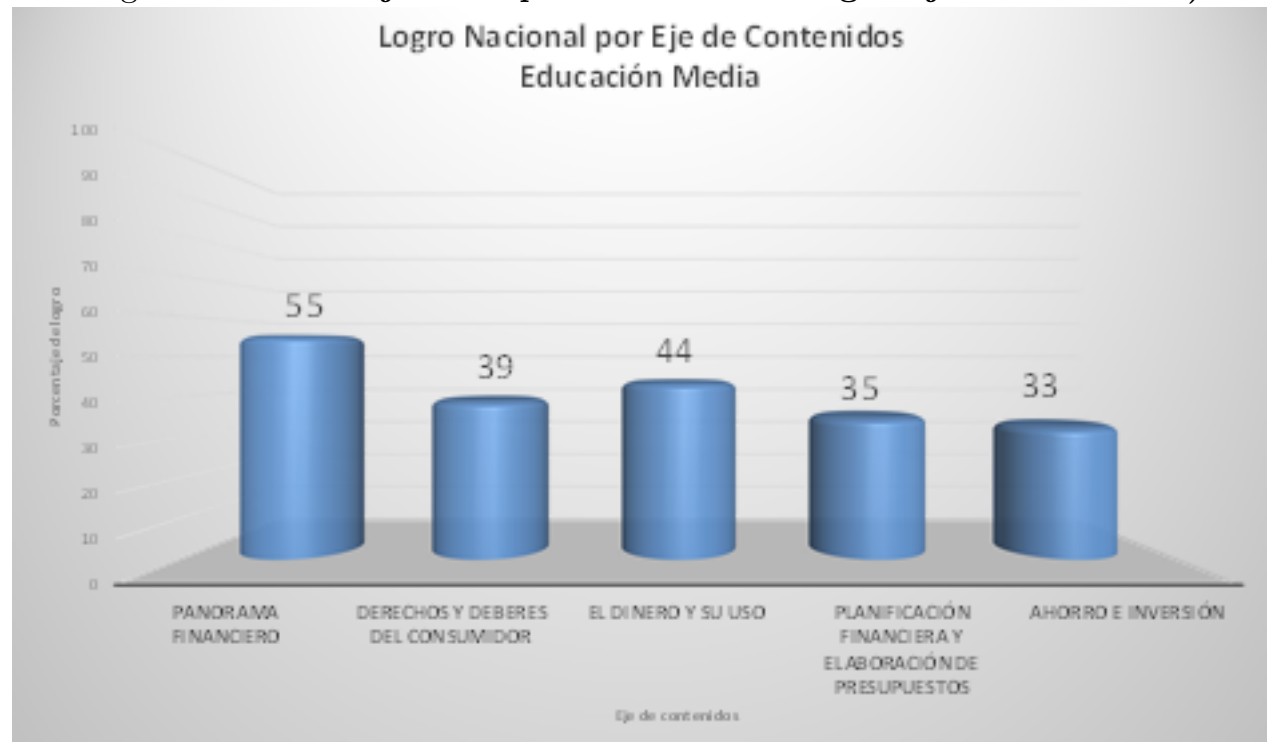

Fuente: Elaboración propia 


\section{Desafíos}

A pesar de los avances, quedan muchos desafíos, tanto para las instituciones públicas como para los establecimientos educacionales. Entre los principales retos a futuro, se encuentra el ampliar la cobertura del programa e incorporarlo como parte de la Estrategia Nacional de Educación Financiera; el participar en los procesos de construcción curricular a nivel nacional del MINEDUC, involucrando la $\mathrm{EF}$ como un conocimiento que debe incluirse en las bases curriculares y planes de estudio, así como el ampliar los contenidos a los niveles iniciales de escolaridad ( $1^{\circ}$ a $6^{\circ}$ básico). Además, el programa se plantea el generar una red de docentes que trabajan la EF en las escuelas, promoviendo la generación de conocimiento en torno al tema y el intercambio de experiencias, el desarrollo de más material de apoyo para los estudiantes y sus familias, y el poder implementar una evaluación de impacto del programa, para observar sus alcances, debilidades, mejoras y efectos.

\section{Referencias}

Denegri, M. (2010). Introducción a la psicología económica. Psicom Editores.

OCDE (2012). Guidelines on financial education in schools. Technical report, Paris. 